\title{
Mounting evidence for immunizing previously infected subjects with a single dose of SARS-CoV-2 vaccine
}

\author{
Gonzalo Perez Marc, Damian Alvarez-Paggi, and Fernando P. Polack
}

Fundación INFANT, Buenos Aires, Argentina

\begin{abstract}
Efforts to best protect the world from SARS-CoV-2 as variants emerge and despite limited vaccine supply are ongoing. One strategy that may maximize vaccine coverage and expedite immunization campaigns involves providing single mRNA vaccine doses to individuals with previous COVID-19. In this issue of the $J C l$, two independent studies, one by Levi and Azzolini et al. and another by Mazzoni and Di Lauria et al., explored vaccine responses in individuals previously infected with the virus. Levi and Azzolini and colleagues used multilinear regression models to correlate exposure and symptoms with antibody response to the vaccine. Mazzoni and Di Lauria and colleagues characterized B cell and T cell kinetics in whole blood after one and two doses of vaccine in health care workers with and without previous infection. Both studies indicated that one vaccine dose may sufficiently protect individuals who have recovered from COVID-19. Implementing a single-dose mRNA vaccine protocol in previously symptomatic individuals may facilitate and expedite immunization campaigns.
\end{abstract}

higher than those in seronegative subjects receiving a two-dose regimen (9). A similar effect was described in 72 health care workers in the United Kingdom, including a subgroup of seronegative volunteers with strong $\mathrm{T}$ cell responses to non-Spike antigens at baseline who exhibited titers of anti-Spike antibody intermediate between naive and seropositive groups 21 to 25 days after receiving the first dose of BNT162b2 (10). SARS-CoV-2-specific T cell responses to Spike peptides were also stronger in individuals with previous natural infection. Importantly, live SARS-CoV-2 neutralization tests in a subset of subjects paralleled the quantitative anti-Spike responses, suggesting that vaccination of previously infected individuals leads to strong neutralizing antibody responses after a single dose of vaccine (10). Immunizing two or more months after symptomatic infection may enhance neutralizing capacity (12). These findings were replicated indirectly by a second study in the United States, using an FDA-approved assay to measure antibodies that block the interaction between the SARS-CoV-2 receptor binding domain and the human host receptor angiotensin-converting enzyme 2 (ACE2) (11).

\section{More doses may not always be better}

Levi and Azzolini et al. and Mazzoni and Di Lauria et al. provide additional context for these observations through the characterization of variables associated with the exponential increase in antibody responses and the description of cellular immune response kinetics after mRNA immunization of previously infected individuals. Levi and Azzolini et al. (7) used multilinear regression models to show that SARSCoV-2 exposure strongly and positively correlated with the antibody response to vaccine, and confirmed an important insight for public health considerations: symptoms matter. Vaccine responses in asymptomatic/paucisymptomatic previously infected subjects were intermediate 
between naive and individuals with classic COVID-19 manifestations. In addition, the data hints at a second important principle in medical practice: more is not always better. A small, in itself anecdotal, subset of previously infected subjects who had experienced florid symptomatology and robust responses to the first dose of vaccine experienced a reduction in antibody titers upon receiving the second dose (7). This observation is supported by the characterization of the kinetics of cellular immunity in Mazzoni and Di Lauria et al. (8).

Mazzoni and Di Lauria and colleagues reported SARS-CoV-2-specific B cell and $\mathrm{T}$ cell kinetics in whole blood after one and two doses of vaccine in health care workers with and without previous infection. In previously infected, symptomatic individuals immunized with the first dose of BNT162b2 vaccine, Spike-protein reactive B cells increased steadily until, with the second inoculation, the effect triggered by the immunogen reverted and specific B cells began to decrease. Additionally, no improvement in the $\mathrm{CD} 4^{+} \mathrm{CD} 154^{+}$, cytokine-positive $\mathrm{T}$ cell population was observed after the second dose. The brisk reactivation of cellular immunity after a single dose of mRNA vaccine in previously infected, symptomatic subjects reported by Mazzoni and Di Lauria and colleagues explains the anamnestic antibody response now evident in all these related publications (8).

\section{Remaining questions}

Many questions emerge from these evolving observations. Obviously, we wonder how long responses to one single dose of mRNA vaccine in previously infected individuals or against two doses in the entire population will last (13). Moreover, many regions of the world, particularly those in greatest need for vaccine supply, are receiving products based on strategies other than mRNA and we must rapidly learn whether these findings are replicated with other constructs (14). We may, in fact, be witnessing the first chapter of a complex, upcoming jigsaw puzzle of vaccine combinations and their effects on the immune response of people living in many countries with intermittent and varied vaccine menus.

A critical question that remains unanswered is whether a single dose of mRNA BNT162b2 vaccine will suffice in previ- ously infected, symptomatic subjects to protect against new SARS-CoV-2 variants, such as B.1.351. Neutralizing activity in convalescent sera from individuals infected early in the pandemic with SARS-CoV-2 is worrisomely low against B.1.351. Further, recent data suggest that a single dose of mRNA vaccine in naive subjects may be less effective against B.1.351 and other variants (15-18). If antibody titers fully account for the poorly protective responses against new SARS-CoV-2 variants in convalescent sera and after a single-dose mRNA immunization in naive individuals, the strong anamnestic response elicited by mRNA vaccines in previously infected subjects should solve the problem. However, if factors such as epitope specificity and antibody affinity in responses triggered by different variants matter, an original antigenic sin phenomenon after WT infection could hamper the anamnestic antibody response after a single dose of vaccine, making a second dose necessary. Eventually, infections with new variants may contribute to the expansion and protective breadth of antibodies and memory B cells in convalescent sera (19). Finally, immunized individuals with prior symptomatic illness and high titers of neutralizing antibody against SARS-CoV-2 may make excellent candidates to expand the pool of donors of high-titer convalescent plasma for early prevention of severe COVID-19 $(20,21)$. People in countries at the end of the receiving line for vaccines need temporary solutions to mitigate this tragedy until all have access to long-term protection. Optimizing this process through focused campaigns in populations sensitized by prior personal experience and with high antibody titers should help.

\section{Conclusions}

The studies described in this Commentary are small, but the core concepts in all of them coincide despite measuring different components of the immune response with different assays in different countries. Follow up in all of them has been limited, although every intervention is novel and has been monitored for a handful of months at best during this pandemic. This said, nothing to date suggests that recovered, symptomatic subjects would benefit from a second dose of vaccine. The immune status of the COVID-19 patient population at the time of vaccination is evidently different from that of naive (or asymptomatically infected) volunteers who participated in the original trials $(2,3)$.

Emergence of new SARS-CoV-2 variants is starting to threaten our pool of vaccines, as data suggest that not every immunogen will work equally well against the variants over time $(22,23)$. This concern is greater for vaccines available in developing countries. Most clinical decisions are not simple and evidence is often limited, but immunizing individuals previously symptomatic due to COVID-19 with a single dose of mRNA vaccine deserves serious consideration.

Address correspondence to: Fernando P. Polack, Fundacion INFANT, Gavilan 94, (1406) Buenos Aires, Argentina. Phone: 5411.4632.5781; Email address: fpolack@ infant.org.ar.

1. Johns Hopkins University. COVID-19 Dashboard. https://coronavirus.jhu.edu/map.html. Updated May 21, 2021. Accessed May 21, 2021.

2. Polack FP, et al. Safety and efficacy of the BNT162b2 mRNA Covid-19 vaccine. $N$ Engl J Med. 2020;383(27):2603-2615.

3. Baden LR, et al. Efficacy and safety of the mRNA-1273 SARS-CoV-2 vaccine. $N$ Engl JMed. 2021;384(5):403-416.

4. Voysey M, et al. Safety and efficacy of the ChAdOx1 nCoV-19 vaccine (AZD1222) against SARS-CoV-2: an interim analysis of four randomised controlled trials in Brazil, South Africa, and the UK. Lancet. 2021;397(10269):99-111.

5. Logunov DY, et al. Safety and efficacy of an rAd26 and rAd5 vector-based heterologous prime-boost COVID-19 vaccine: an interim analysis of a randomised controlled phase 3 trial in Russia. Lancet. 2021;397(10275):671-681.

6. Sadoff J, et al. Interim results of a phase 1-2a trial of Ad26.COV2.S Covid-19 vaccine. $N$ Engl J Med. 2021;384(19):1824-1835.

7. Levi R, et al. One dose of SARS-CoV-2 vaccine exponentially increases antibodies in individuals who have recovered from symptomatic COVID-19. J Clin Invest. 2021;131(12):e149154.

8. Mazzoni A, et al. First-dose mRNA vaccination is sufficient to reactivate immunological memory to SARS-CoV-2 in subjects who have recovered from COVID-19 [published online May 3, 2021]. JClin Invest. https://doi.org/10.1172/JCI149150.

9. Krammer F, et al. Antibody responses in seropositive persons after a single dose of SARS-CoV-2 mRNA vaccine. $N$ EnglJ Med. 2021;384(14):1372-1374.

10. Prendecki M, et al. Effect of previous SARSCoV-2 infection on humoral and T- cell responses to single-dose BNT162b2 vaccine. Lancet. 2021;397(10280):1178-1181.

11. Bradley T, et al. Antibody responses after a single dose of SARS-CoV-2 mRNA vaccine. $N$ EnglJ 
Med. 2021;384(20):1959-1961.

12. Anichini G, et al. SARS-CoV-2 antibody response in persons with past natural infection [published online April 14, 2021]. N EnglJ Med. https://doi. org/10.1056/nejmc2103825.

13. Doria-Rose N, et al. Antibody persistence through 6 months after the second dose of mRNA-1273 vaccine for Covid-19 [published online April 6, 2021]. N Engl J Med. https://doi. org/10.1056/nejmc2103916.

14. Chahla RE, et al. Past SARS-CoV-2 infection elicits a strong immune response after a single vaccine dose [preprint]. https://doi.org/10.11 01/2021.03.14.21253039. Posted on medRxiv March 24, 2021.

15. Hacisuleyman E, et al. Vaccine breakthrough infections with SARS-CoV-2 variants [published online April 21, 2021]. N Engl J Med. https://doi. org/10.1056/nejmoa2105000.

16. Wang GL, et al. Susceptibility of circulating SARS-CoV-2 variants to neutralization [published online April 6, 2021]. N EnglJMed. https://doi.org/10.1056/nejmc2103022.

17. Liu $Y$, et al. Neutralizing activity of BNT162b2-elicited serum. $N$ Engl J Med. 2021;384(15):1466-1468.

18. Wu K, et al. Serum neutralizing activity elicited by mRNA-1273 vaccine. $N$ Engl J Med. 2021;384(15):1468-1470.

19. Moyo-Gwete T, et al. Cross-reactive neutralizing antibody responses elicited by SARS-CoV-2 501Y.V2 (B.1.351) [published online April 7,
2021]. N EnglJMed. https://doi.org/10.1056/ nejmc2104192.

20. Joyner MJ, et al. Convalescent plasma antibody levels and the risk of death from Covid-19. NEngl JMed. 2021;384(11):1015-1027.

21. Libster R, et al. Early high-titer plasma therapy to prevent severe Covid-19 in older adults. NEnglJMed. 2021;384(7):610-618.

22. Ikegame $\mathrm{S}$, et al. Qualitatively distinct modes of Sputnik V vaccine-neutralization escape by SARS-CoV-2 Spike variants [preprint]. https:// doi.org/10.1101/2021.03.31.21254660. Posted on medRxiv April 3, 2021.

23. Madhi SA, et al. Efficacy of the ChAdOx $1 \mathrm{nCoV}$ 19 Covid-19 vaccine against the B.1.351 variant. N Engl JMed. 2021;384(20):1885-1898. 